\title{
ARTIGOS
}

\section{REAÇÃO DE GENÓTIPOS DE Vigna unguiculata À SARNA E EFEITO DA DOENÇA SOBRE COMPONENTES DE PRODUÇÃO}

\author{
PAULO D. BARRETO ${ }^{1}$, ANTÔNIO A. SANTOS ${ }^{1}$, JÚLIO C. VIDAL ${ }^{1}$, MARY A. W. QUINDERÉ 2 \\ \& MARIA F. P. SÁ ${ }^{2}$ \\ ${ }^{1}$ Embrapa Agroindústria Tropical, Rua Dra. Sara Mesquita, 2270, Pici, CEP 60511-110, Fortaleza, CE, \\ e-mail:apoliano@ cnpat.embrapa.br; ${ }^{2}$ Empresa de Pesquisa Agropecuária do Ceará - EPACE (extinta em 1998)
}

(Aceito para publicação em 05/12/2000)

Autor para correspondência: Antônio Apoliano dos Santos

BARRETO, P.D., SANTOS, A.A., VIDAL, J.C., QUINDERÉ, M.A.W. \& SÁ, M.F.P. Reação de genótipos de Vigna unguiculata à sarna e efeito da doença sobre componentes de produção. Fitopatologia Brasileira 26:5-9. 2001.

\section{RESUMO}

O fungo Elsinoe phaseoli, agente causal da sarna, tem sido responsável por sérios prejuízos aos produtores de feijão-de-corda (Vigna unguiculata), em particular, nos cultivos que se estabelecem em regiões montanhosas e na época das chuvas, no Ceará. O presente trabalho objetivou, além de identificar fontes de resistência à sarna, avaliar os efeitos da doença sobre o rendimento agrícola e seus componentes, a qualidade do grão e o ciclo da cultura. Num ensaio delineado em blocos ao acaso, com quatro repetições, instalado em Tianguá, CE, sob regime de irrigação e em sequeiro, por dois anos: 1995 e 1996, 16 genótipos foram avaliados quanto a variáveis relacionadas ao ciclo, rendimento de grãos e sintomas de sarna. Procederam-se análises quanto à variância, comparações entre médias dos tratamentos, correlação e regressão. Constatou-se que as plantas suscetíveis à sarna sofrem seus efeitos negativos sobre ciclo e produção de grãos; as cultivares e linhagens avaliadas apresentaram variabilidade quanto à reação à doença. A cultivar EPACE V-96, pela produtividade, precocidade e grau de resistência à sarna, é a mais indicada como genitor, visando o melhoramento genético do feijão-de-corda para o ambiente da Ibiapaba, CE.

Palavras-chave: feijão-de-corda, caupi, melhoramento, doença, fungo, Elsinoe phaseoli.

\section{ABSTRACT \\ Reaction of Vigna unguiculata genotypes to scab}

The fungus Elsinoe phaseoli is the scab agent and has been causing serious damages to the cowpea (Vigna unguiculata), crop, mainly in the rainy period of Ceara State's mountainous areas. The present work aimed to identify genetic resistance sources and to evaluate the disease effects on the yield and its components, the quality of the grain and the crop cycle. To do this, in 1995 and 1996, 16 genotypes were evaluated in two experiments. One at the rainy period as a rain-fed crop and the other at the dry period as a irrigated crop, in a randomized experimental design with four replications in Tianguá County, State of Ceará. Based on statistical analysis and evaluation of the variances, comparing the treatment averages, the correlation and the regression, it was verified that: in the scab susceptible plants both the cycle and the yield are negatively affected by the disease; all evaluated genotypes have variability in relation to the disease reaction; and the EPACE V-96 cultivar performed better and so can be utilized as parental in a breeding program.

\section{INTRODUÇÃO}

O cultivo do feijão-de-corda [Vigna unguiculata (L.) Walp.], constitui atividade agrícola tradicional no Estado do Ceará, face a sua importância econômico-social. Em conseqüência, é cultivado na totalidade dos municípios (Anuário, 1996), em ambientes cujos fatores, especialmente aqueles relacionados ao solo e ao clima, experimentam consideráveis variações. Tais variações exercem ações distintas sobre a planta, entre elas a ocorrência de doenças favorecidas por cada condição ambiental. Sob altitudes elevadas, como na Serra da Ibiapaba, as condições de temperatura e umidade, que ocorrem na época chuvosa, favorecem a infecção por diversos fungos patogênicos. Segundo Torres Filho \& Sá (1994), a cultura, que representa a principal opção econômica para os pequenos agricultores da região, em particular para os que a exploram na região ecológica do "Carrasco" vem sofrendo prejuízos nos últimos 
anos, devido à elevada incidência e severidade da sarna causada pelo fungo Elsinoe phaseoli Jenkins.

Em nível mundial, a doença é responsável por graves danos no norte da Nigéria, na América Central e no Suriname (Singh \& Allen, 1979). De acordo com Rios (1988), sob condições propícias, o problema acentua-se porque o patógeno é transmitido, em altas taxas, através das sementes, e sobrevive em restos de culturas, constituindo estas as principais fontes de infecção primária. Os sintomas provocados pela sarna são muito severos e logo no início da produção, as perdas são grandes em decorrência do abortamento das vagens que se tornam torcidas ou curvas, caem ou os grãos não se desenvolvem (Rios, 1990). Tem-se observado que, mesmo quando as perdas quantitativas não são mensuradas, os grãos são afetados, qualitativamente, quer pela a redução no tamanho, quer pela presença de manchas escuras ocasionadas pelo aprofundamento das lesões nas vagens.

Como alternativa ao controle químico com suas restrições de natureza ecológica, os pesquisadores têm procurado solucionar o problema mediante emprego de resistência genética (Rios \& Watt, 1980). Rios (1988) indica que numerosas linhagens dentro do cruzamento CNCx 249, foram selecionadas para resistência à sarna. No entanto, conforme revisão de Emechebe \& Florini (1997), a resistência genética vem a ser solução específica de cada local, pois a linhagem TVx 3236, que é resistente à sarna na Nigéria, é suscetível em Burkina Faso. No Estado do Ceará, Sousa et al. (1992) avaliaram, em Tianguá, 22 linhagens, identificando materiais resultantes dos cruzamentos $\operatorname{CNCx}(\mathrm{s})$ '242', '249', e 252 que não apresentaram sintomas da doença. Com o mesmo intuito Torres Filho \& Sá (1994), em novas introduções, relacionaram como imunes linhagens dos cruzamentos $\mathrm{CNCx}(\mathrm{s})$ '664', '673' e '676'. Seguem-se na mesma linha, os trabalhos de Barreto et al. (1996a), Barreto et al. (1996b), Torres Filho \& Sá (1996), Torres Filho \& Sousa (1996).

Utilizando germoplasma desenvolvido na Embrapa Centro Nacional de Pesquisa de Arroz e Feijão (CNPAF) e Centro de Pesquisa Agropecuária do Meio Norte (CPAMN), o presente trabalho objetivou, além da identificação de fontes de resistência à sarna, examinar sob que condições o fungo infeta o feijão-de-corda no Estado do Ceará, bem como os efeitos da doença sobre o rendimento agrícola e seus componentes, sobre a qualidade do grão e sobre o ciclo da cultura.

\section{MATERIAL E MÉTODOS}

Um ensaio em rede foi instalado em seis municípios, sendo para este trabalho extraídos apenas os dados obtidos em Tianguá - CE, em razão de apenas neste local ocorrer infecção por E. phaseoli. Foram avaliados 16 genótipos, sendo: três linhagens (seleção individual na $6^{\mathrm{a}}$ geração póscruzamento) oriundos da Embrapa - CNPAF (CNCx 923-8F, CNCx 925-1F e CNCx 928-6F), nove linhagens (selecionadas na $5^{\text {a }}$ geração) procedentes da Embrapa - CPAMN (TE90178-4E, TE90-179-10E, TE90-180-3E, TE90-180-9E, TE90180-12E, TE90-180-13E, TE90-180-15E, TE90-180-16E e TE90-180-18E), três cultivares usadas como testemunhas melhoradas (EPACE 10, EPACE 11 e EPACE V-96) e, finalmente, uma testemunha local - "Moita de Crateús". As observações foram feitas na Estação Experimental da extinta Empresa de Pesquisa Agropecuária do Ceará - EPACE, localizada no município de Tianguá-CE.

O ensaio foi conduzido por dois anos: 1995 e 1996, nas condições de sequeiro e irrigado e, em cada situação, dois experimentos: um submetido ao controle de pragas e outro, não. Adotou-se o delineamento de blocos ao acaso, com quatro repetições. Cada unidade experimental constituiu-se de quatro fileiras de 5,00 m espaçadas de $0,70 \mathrm{~m}$ por $0,30 \mathrm{~m}$ entre covas, com duas plantas após o desbaste e área útil, formada pelas duas fileiras centrais. Na condução dos experimentos empregaram-se práticas culturais normalmente adotadas no manejo da cultura.

Coletaram-se dados para produção de grãos (kg/ha) e seus componentes: comprimento de vagens (média, em cm, obtida da medida de 10 vagens), número de grãos por vagens (média obtida a partir da contagem dos grãos de 10 vagens retiradas aleatoriamente de cada parcela) e peso de 100 grãos (em gramas); variáveis ligadas ao ciclo: floração e maturação inicial (dias transcorridos desde o plantio até a ocorrência das primeiras vagens maduras) e nível de infecção por $E$. phaseoli, baseado em escores atribuídos a partir de observação visual em campo, sob infecção natural, variando de 1 a 5 , onde: $1=$ ausência de sintomas; $2=$ menos de $25 \%$ da área lesionada; 3 = de 25 a $50 \%$ da área lesionada; $4=$ de 51 a $75 \%$ da área lesionada e; $5=$ mais de $75 \%$ da área lesionada. A partir das 512 observações obtidas dos 16 genótipos submetidos às diferentes condições impostas, foram processadas as análises de variância, de correlação e de regressão.

\section{RESULTADOS E DISCUSSÃO}

A análise de variância (Tabela 1) revela significância dos efeitos principais (ano, suprimento hídrico, controle de pragas e genótipos) sobre todas as variáveis estudadas, com exceção do controle de pragas que, conforme esperado, não teve qualquer influência sobre o nível de infecção pela sarna. Por outro lado, constatou-se que as interações, entre o suprimento hídrico com ano e com genótipos (Tabela 1) foram, estatisticamente significativas, não apenas para a intensidade dos sintomas de sarna, mas também para as variáveis produção de grãos e seus componentes (comprimento de vagens, número de grãos por vagem e peso por 100 grãos). Isto indica que tanto as características do ambiente sob sequeiro podem variar de ano a ano, como mais acentuadas ainda são as diferenças entre as condições de sequeiro e de irrigação, que influem de modo diferenciado sobre o comportamento dos genótipos. Entre os materiais avaliados, a linhagem CNCx 928-6F e a cultivar EPACE V-96, reafirmando resultados obtidos por 
Reação de genótipos de Vigna unguiculata à sarna e efeito...

TABELA 1 - Médias, variâncias, coeficientes de variação e valores de "F" obtidos para as variáveis sarna, floração inicial, maturação inicial, comprimento de vagens, número de grãos por vagem, peso por 100 grãos e rendimento de grãos de feijão-de-corda (Vigna unguiculata), no município de Tianguá, CE, sob regimes de sequeiro e irrigado, nos anos 1995 e 1996

\begin{tabular}{|c|c|c|c|c|c|c|c|}
\hline \multirow[b]{2}{*}{ Fonte de variação } & \multicolumn{7}{|c|}{ Variáveis $^{1}$} \\
\hline & Sarna & $\begin{array}{c}\text { Floração } \\
\text { inicial (dias) }\end{array}$ & $\begin{array}{c}\text { Maturação } \\
\text { inicial (dias) }\end{array}$ & $\begin{array}{c}\text { Comprimento de } \\
\text { vagens }(\mathrm{cm})\end{array}$ & $\begin{array}{c}\mathrm{N}^{0} \text { de } \\
\text { grãos/vagem } \\
\end{array}$ & $\begin{array}{c}\text { Peso/100 grãos } \\
(\mathrm{g})\end{array}$ & Rendimento (kg/ha) \\
\hline \multicolumn{8}{|l|}{ Genótipo } \\
\hline CNCx 928-6F & $1,16 \mathrm{bc}$ & $45,66 \mathrm{de}$ & $65,47 \mathrm{~d}$ & $18,18 \mathrm{abc}$ & 14,11 abcdef & $14,56 \mathrm{e}$ & $1.136,60 \mathrm{ab}$ \\
\hline CNCx 925-1F & $1,56 \mathrm{ab}$ & $47,69 \mathrm{bcd}$ & $68,72 \mathrm{bc}$ & $17,81 \mathrm{abc}$ & 14,38 abcd & $16,31 \mathrm{bcd}$ & $1.142,20 \mathrm{ab}$ \\
\hline TE90-180-13E & $1,56 \mathrm{ab}$ & $48,75 \mathrm{abc}$ & $68,88 \mathrm{bc}$ & $18,49 \mathrm{ab}$ & 14,39 abcd & $15,81 \mathrm{cde}$ & $891,60 \mathrm{ab}$ \\
\hline TE90-180-18E & $1,59 \mathrm{a}$ & 49,22 abc & $69,75 \mathrm{ab}$ & 16,09 de & 13,74 abcdef & $15,16 \mathrm{de}$ & $910,30 \mathrm{ab}$ \\
\hline TE90-179-10E & $1,63 \mathrm{a}$ & $50,44 \mathrm{a}$ & $71,03 \mathrm{ab}$ & 17,15 bcde & 14,40 abcd & 15,37 cde & $978,90 \mathrm{ab}$ \\
\hline TE90-180-3E & $1,66 \mathrm{a}$ & $50,06 \mathrm{ab}$ & $70,13 \mathrm{ab}$ & 17,44 bcde & $12,63 \mathrm{f}$ & $16,22 \mathrm{bcd}$ & $809,40 \mathrm{~b}$ \\
\hline Moita de Crateus & $1,66 \mathrm{a}$ & $49,03 \mathrm{abc}$ & $69,41 \mathrm{ab}$ & 17,56 abcd & 14,22 abcde & $16,23 \mathrm{bcd}$ & $789,80 \mathrm{~b}$ \\
\hline EPACE 10 & $1,72 \mathrm{a}$ & $49,91 \mathrm{ab}$ & $71,50 \mathrm{a}$ & 17,49 abcde & $14,44 \mathrm{abc}$ & $17,23 \mathrm{ab}$ & $1.241,30 \mathrm{a}$ \\
\hline EPACE-11 & $1,72 \mathrm{a}$ & $48,63 \mathrm{abc}$ & $68,78 \mathrm{bc}$ & $19,15 \mathrm{a}$ & $14,88 \mathrm{a}$ & $18,23 \mathrm{a}$ & $1.097,10 \mathrm{ab}$ \\
\hline TE90-180-16E & $1,72 \mathrm{a}$ & $49,94 \mathrm{ab}$ & $70,34 \mathrm{ab}$ & 16,63 cde & 13,28 cdef & $16,54 \mathrm{bc}$ & $1.131,90 \mathrm{ab}$ \\
\hline TE90-180-12E & $1,75 \mathrm{a}$ & $49,69 \mathrm{ab}$ & $70,22 \mathrm{ab}$ & $16,05 \mathrm{de}$ & 12,83 ef & 15,42 cde & $908,20 \mathrm{ab}$ \\
\hline TE90-180-9E & $1,78 \mathrm{a}$ & 50,84 a & $71,03 \mathrm{ab}$ & $16,67 \mathrm{cde}$ & 12,87 ef & $16,30 \mathrm{bcd}$ & $836,90 \mathrm{~b}$ \\
\hline CNCx $923-8 \mathrm{~F}$ & $1,88 \mathrm{a}$ & $46,72 \mathrm{~cd}$ & $66,63 \mathrm{~cd}$ & 17,10 bcde & 14,27 abcde & $15,14 \mathrm{de}$ & $1.075,90 \mathrm{ab}$ \\
\hline \multicolumn{8}{|l|}{ Controle de pragas } \\
\hline Sem controle & $1,61 \mathrm{a}$ & $49,17 \mathrm{a}$ & $69,11 \mathrm{a}$ & $17,23 \mathrm{a}$ & $13,64 \mathrm{~b}$ & $16,02 \mathrm{a}$ & 983,16 a \\
\hline Com controle & 1,66 a & $48,19 \mathrm{~b}$ & $69,24 \mathrm{a}$ & $17,23 \mathrm{a}$ & $14,05 \mathrm{a}$ & $15,69 \mathrm{~b}$ & $1.043,10 \mathrm{a}$ \\
\hline \multicolumn{8}{|l|}{ Suprimento hídrico } \\
\hline Sequeiro & $2,27 \mathrm{a}$ & $48,81 \mathrm{a}$ & 69,89 a & $18,51 \mathrm{a}$ & $13,39 \mathrm{~b}$ & $13,86 \mathrm{~b}$ & $549,50 \mathrm{~b}$ \\
\hline Irrigado & $1,00 \mathrm{~b}$ & $48,55 \mathrm{a}$ & $68,45 \mathrm{~b}$ & $15,96 \mathrm{~b}$ & $14,30 \mathrm{a}$ & $17,84 \mathrm{a}$ & $1.476,77 \mathrm{a}$ \\
\hline \multicolumn{8}{|l|}{ Ano } \\
\hline 1995 & $1,59 \mathrm{~b}$ & 50,66 a & $70,20 \mathrm{a}$ & $16,95 \mathrm{~b}$ & $13,38 \mathrm{~b}$ & $14,94 \mathrm{~b}$ & $793,06 \mathrm{~b}$ \\
\hline 1996 & $1,68 \mathrm{a}$ & $46,71 \mathrm{~b}$ & $68,15 \mathrm{~b}$ & $17,52 \mathrm{a}$ & $14,31 \mathrm{a}$ & 16,76 a & $1.233,20 \mathrm{a}$ \\
\hline$\sigma^{2}$ & 0,71 & 0,58 & 0,58 & 0,51 & 0,40 & 0,78 & 0,71 \\
\hline C.V. $(\%)$ & 31,02 & 6,33 & 4,00 & 11,28 & 12,43 & 9,85 & 40,37 \\
\hline $\mathbf{Q M E} \mathbf{E}^{-1 / 2}$ & 0,50 & 3,08 & 2,76 & 1,94 & 1,72 & 1,56 & 408,99 \\
\hline F-Ano & $4,39^{2}$ & $210,12^{3}$ & $70,43^{3}$ & $10,97^{3}$ & $36,71^{3}$ & $172,85^{3}$ & $148,24^{3}$ \\
\hline F-Suprimento hídrico & $799,18^{3}$ & $0,87 \mathrm{~ns}$ & $34,79^{3}$ & $221,54^{3}$ & $35,96^{3}$ & $834,69^{3}$ & $657,95^{3}$ \\
\hline F-Controle de pragas & $1,10 \mathrm{~ns}$ & $12,95^{3}$ & $0,28 \mathrm{~ns}$ & $0,00 \mathrm{~ns}$ & $7,24^{3}$ & $5,78^{2}$ & $2,75 \mathrm{~ns}$ \\
\hline F-Genótipo & $5,66^{3}$ & $13,29^{3}$ & $14,03^{3}$ & $6,82^{3}$ & $6,06^{3}$ & $12,79^{3}$ & $4,16^{3}$ \\
\hline $\mathrm{F}-\mathrm{A} * \mathrm{SH}$ & $4,39^{2}$ & $0,25 \mathrm{~ns}$ & $64,14^{3}$ & $0,99 \mathrm{~ns}$ & $6,70^{2}$ & $149,84^{3}$ & $1,15 \mathrm{~ns}$ \\
\hline $\mathrm{F}-\mathrm{A} * \mathrm{CP}$ & $0,12 \mathrm{~ns}$ & $15,32^{3}$ & $44,01^{3}$ & $0,26 \mathrm{~ns}$ & $8,33^{3}$ & $0,18 \mathrm{~ns}$ & $1,33 \mathrm{~ns}$ \\
\hline $\mathrm{F}-\mathrm{A} * \mathrm{G}$ & $1,32 \mathrm{~ns}$ & $3,64^{3}$ & $2,66^{3}$ & $1,23 \mathrm{~ns}$ & $1,13 \mathrm{~ns}$ & $2,84^{3}$ & $1,73^{2}$ \\
\hline $\mathrm{F}-\mathrm{SH}{ }^{*} \mathrm{CP}$ & $1,10 \mathrm{~ns}$ & $10,97^{3}$ & $0,66 \mathrm{~ns}$ & $1,40 \mathrm{~ns}$ & $1,92 \mathrm{~ns}$ & $18,31^{3}$ & $2,95 \mathrm{~ns}$ \\
\hline F-SH*G & $5,66^{3}$ & $1,33 \mathrm{~ns}$ & $4,33^{3}$ & $2,05^{2}$ & $1,24 \mathrm{~ns}$ & $2,08^{2}$ & $2,12^{3}$ \\
\hline $\mathrm{F}-\mathrm{CP} * \mathrm{G}$ & $0,66 \mathrm{~ns}$ & $0,82 \mathrm{~ns}$ & $1,39 \mathrm{~ns}$ & $0,87 \mathrm{~ns}$ & $1,70^{2}$ & $2,01^{2}$ & $1,50 \mathrm{~ns}$ \\
\hline $\mathrm{F}-\mathrm{A} * \mathrm{SH} \mathrm{H}^{*} \mathrm{G}$ & $1,32 \mathrm{~ns}$ & $0,79 \mathrm{~ns}$ & $1,00 \mathrm{~ns}$ & $1,03 \mathrm{~ns}$ & $0,72 \mathrm{~ns}$ & $1,19 \mathrm{~ns}$ & $1,73^{2}$ \\
\hline $\mathrm{F}-\mathrm{A} * \mathrm{CP} * \mathrm{G}$ & $1,14 \mathrm{~ns}$ & $1,37 \mathrm{~ns}$ & $1,02 \mathrm{~ns}$ & $1,12 \mathrm{~ns}$ & $0,89 \mathrm{~ns}$ & $0,55 \mathrm{~ns}$ & $1,60 \mathrm{~ns}$ \\
\hline
\end{tabular}

${ }^{1}$ Médias seguidas da mesma letra não são significativamente diferentes entre si (Tukey, 5\%)

${ }^{2}$ Significativo ao nível de $5 \%$ de probabilidade

${ }^{3}$ Significativo ao nível de $1 \%$ de probabilidade

ns- Não significativo

Barreto et al. (1994), Barreto et al. (1996a) e Barreto et al. (1996b), apresentaram o melhor comportamento ante a infecção por E. phaseoli. O controle de pragas, para a região cujo clima desfavorece a infestação da cultura por insetos, não influiu sobre as médias obtidas para as variáveis mais importantes: sarna, maturação e rendimento de grãos. Já quanto ao ano e ao meio de suprimento hídrico, médias comparadas pelo teste Tukey (5\%) diferenciaram-se para a maioria das variáveis, destacando-se a ocorrência de sarna dentro de suprimento hídrico, em que não se registra a doença sob condição natural em regime de irrigação. Provavelmente este fato tenha contribuído para acentuar consideráveis diferenças em tamanhos de grãos ( $\cong 4 \mathrm{~g} / 100$ grãos) e rendimentos (cerca de $1.000 \mathrm{~kg} / \mathrm{ha}$ ) entre os dois sistemas. No entanto, os 
dados indicam que é possível cultivar feijão-de-corda na região da Ibiapaba, CE, explorando-se a variabilidade genética disponível quanto à resistência à sarna. A cultivar EPACE V96 (CNCx 698-128G), mesmo rejeitada comercialmente devido ao tamanho do grão, poderia ser utilizada como genitora não apenas por ser portadora de resistência genética ao fungo, mas também, conforme Barreto et al. (1996b), pelo elevado potencial de produção e elevada adaptabilidade ambiental.

A matriz de correlações estabelecida entre as diferentes variáveis estudadas (Tabela 2) mostra que, com algumas exceções, foram significativamente correlacionadas entre si. A sarna, foco deste trabalho, influiu sobre todas as demais variáveis: aumentando o período para início de florescimento

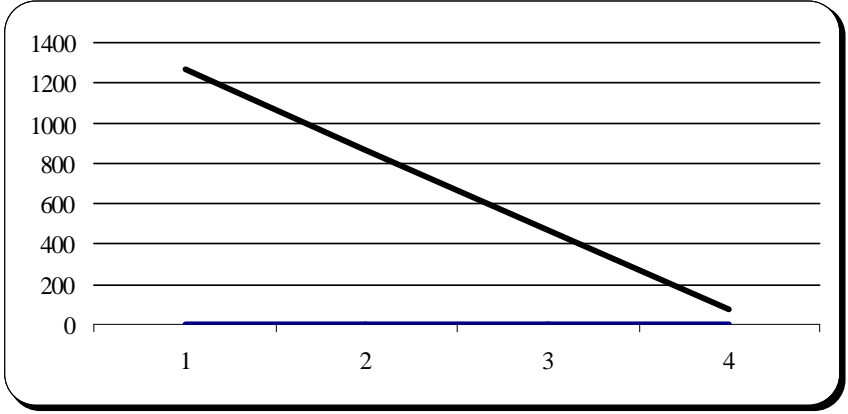

FIG. 1 - Rendimento de grãos (kg/ha) de feijão-de-corda (Vigna unguiculata L.) estimado em função do nível de infecção das plantas por Elsinoe phaseoli.

TABELA 2 - Matriz de correlações (Pearson, probabilidade $>|R|$, pressupondo Ho: $R$ ho=0, $N=512$ ) entre as variáveis sarna, floração inicial, maturação inicial, comprimento de vagens, $n^{0}$ de grãos por vagem, peso de 100 grãos e rendimento (kg/ha) de feijão-de-corda (Vigna unguiculata $\mathrm{L}$.)

\begin{tabular}{|c|c|c|c|c|c|c|}
\hline Variável & $\begin{array}{c}\text { Floração inicial } \\
\text { (dias) }\end{array}$ & $\begin{array}{c}\text { Maturação } \\
\text { inicial (dias) }\end{array}$ & $\begin{array}{l}\text { Comprimento de } \\
\text { vagens }(\mathrm{cm})\end{array}$ & $\mathrm{N}^{0}$ de grãos/vagem & $\begin{array}{c}\text { Peso/100 grãos } \\
\text { (g) }\end{array}$ & $\begin{array}{c}\text { Rendimento } \\
\text { (kg/ha) }\end{array}$ \\
\hline Sarna & $0,08714 *$ & $0,21764 * *$ & $-0,39718 * *$ & $-0,13975 * *$ & $-0,43021 * *$ & $-0,49867 * *$ \\
\hline Floração inicial (dias) & & $0,46331 * *$ & $-0,02216 \mathrm{~ns}$ & $-0,18431 * *$ & $-0,03909 \mathrm{~ns}$ & $-0,33635 * *$ \\
\hline Comprimento de vagens $(\mathrm{cm})$ & & & & $0,44345 * *$ & $0,53660 * *$ & $0,35820 * *$ \\
\hline $\mathrm{N}^{\mathrm{o}}$ de grãos/vagem & & & & & $0,28567 * *$ & $0,32711 * *$ \\
\hline Peso/100 grãos (g) & & & & & & $0,49840 * *$ \\
\hline
\end{tabular}

*Significativo ao nível de $5 \%$ de probabilidade.

**Significativo ao nível de $1 \%$ de probabilidade.

ns- Não significativo.

TABELA 3 - Regressão linear para diferentes variáveis em função do nível severidade dos sintomas de sarna nas plantas de feijão-de-corda (Vigna unguiculata L.)

\begin{tabular}{|c|c|c|c|c|c|c|c|}
\hline \multirow{2}{*}{ Variável dependente } & \multirow{2}{*}{ Valor de 'F' } & \multirow{2}{*}{$\mathrm{QME}^{-1 / 2}$} & \multirow{2}{*}{ Média } & \multirow{2}{*}{$\begin{array}{l}\text { C.V. } \\
(\%)\end{array}$} & \multicolumn{2}{|c|}{ T para Ho: Parametro $=0$} & \multirow{2}{*}{ Equação linear $(y=a+b x)$} \\
\hline & & & & & $\mathbf{a}$ & Sarna (b) & \\
\hline Floração inicial (dias) & $3,90 *$ & 4,27 & 48,68 & 8,76 & $118,14 * *$ & $1,98 *$ & $\mathrm{~F}=47,97+0,44 \mathrm{~S}$ \\
\hline Maturação inicial (dias) & $25,35 * *$ & 3,77 & 69,17 & 5,45 & $188,24 * *$ & $5,04 * *$ & $\mathrm{M}=67,57+0,98 \mathrm{~S}$ \\
\hline Comp. vagem $(\mathrm{cm})$ & $95,52 * *$ & 2,29 & 17,23 & 13,33 & $87,41 * *$ & $-9,77 * *$ & $\mathrm{CV}=19,13-1,16 \mathrm{~S}$ \\
\hline $\mathrm{N}^{\mathrm{o}}$ de grãos/vagem & $10,15 * *$ & 1,98 & 13,84 & 14,29 & $76,29 * *$ & $-3,19 * *$ & $\mathrm{NGV}=14,38-0,33 \mathrm{~S}$ \\
\hline Peso/100 grãos (g) & $115,83 * *$ & 2,73 & 15,85 & 17,24 & $70,46 * *$ & $-10,76 * *$ & $\mathrm{P} 100 \mathrm{G}=18,33-1,52 \mathrm{~S}$ \\
\hline Rendimento $(\mathrm{kg} / \mathrm{ha})$ & $168,80 * *$ & 591,94 & $1.013,13$ & 58,43 & $29,49 * *$ & $-12,99 * *$ & REND $=1.661,61-397,16 \mathrm{~S}$ \\
\hline
\end{tabular}

*Significativo ao nível de $5 \%$ de probabilidade.

e para maturação de vagens, reduzindo o comprimento das vagens, o número de grãos por vagem, o peso por 100 grãos e, em consequiência, o rendimento de grãos ( $\mathrm{kg} / \mathrm{ha})$, assim como observado por Rios (1990). O procedimento de regressão entre variáveis mostra que, para o modelo linear $(y=a+b x)$, todos os parâmetros das equações estabelecidas entre cada variável em função dos graus de infecção por sarna nas plantas foram altamente significativos (Tabela 3). Entre as equações, a de maior interesse prático $(\mathrm{R}=1.661,61$ - 397,16S) mostra que, para valores de "S" superiores a 4,18 , a sarna pode explicar a perda total na produção de grãos (Figura 1).

\section{REFERÊNCIAS BIBLIOGRÁFICAS}

ANUÁRIO ESTATÍSTICO DO BRASIL. Rio de Janeiro: IBGE, 1996.

BARRETO, P.D., QUINDERÉ, M.A.W., SANTOS, A.A., VIDAL, J.C. \& SÁ, M.F.P. Melhoramento de caupi para o Estado do Ceará. In: EPACE (Fortaleza, CE). Resultados das atividades de pesquisa alcançados em 1992/93: Resumos. Fortaleza. 1994. p.36.

BARRETO, P.D., QUINDERÉ, M.A.W., SÁ, M.F.P. \& SANTOS, A.A. CNCx 698-128G - Linhagem de caupi, Vigna unguiculata (L.) Walp., com alto potencial de 
produção e adaptabilidade às diferentes condições de cultivo do Ceará. Resumos, $4^{\mathrm{a}}$ Reunião Nacional de Pesquisa de Caupi, Teresina, PI.(Embrapa-CPAMN. Documentos, 18). 1996a, p.83-84.

BARRETO, P.D., QUINDERÉ, M.A.W., SÁ, M.F.P. \& SANTOS, A.A. Comportamento de linhagens de feijãode-corda, Vigna unguiculata (L.) Walp., em quatro municípios do Ceará. Fortaleza: EPACE, Comunicado Técnico, 50. 1996b.

EMECHEBE, A.M. \& FLORINI, D.A. Shoot and pod diseases of cowpea induced by fungi and bacteria. In: SINGH, B.B; MOHAN RAJ, D.R., DASHIELL, K.E. \& JACKAI, L.E.N. (Eds.) Advances in Cowpea Research. Ibadan. IITA/Tsukuba. JIRCAS. 1997. pp. 176-206.

RIOS, G.P. Doenças fúngicas e bacterianas do caupi. In: ARAUJO, J.P.P. de \& WATT, E.E. (Org.) O caupi no Brasil. Brasília. Embrapa/IITA. 1988. cap.19, p.547-589.

RIOS, G.P. Principais doenças do caupi no Brasil. Goiânia. Embrapa - CNPAF. Documentos, 291990.

RIOS, G.P. \& WATT, E.E. Identification de fuentes de resistência a las principales enfermedades de caupi
(Vigna unguiculata (L.) Walp.). Fitopatologia 15:24. 1980. (Suplemento).

SINGH, S.R. \& ALLEN, D.J. Parasitos y enfermidades del caupi. Ibadan. IITA. Manual Series, 2. 1979.

SOUSA, M.C.M.R. de, TORRES FILHO, J. \& SÁ, M. de F.P. Reação de cultivares de caupi, Vigna unguiculata, à sarna, Sphaceloma sp, no planalto da Ibiapaba. In: EPACE (Fortaleza, CE). Resultados das atividades de pesquisa alcançados em 1991: Resumos. Fortaleza, 1992. p.49.

TORRES FILHO, J. \& SÁ, M. de F.P. Seleção de fontes de resistência à sarna em caupi no Planalto da IbiapabaCE. In: EPACE (Fortaleza, CE). Resultados das atividades de pesquisa alcançados em 1992/93: Resumos. Fortaleza, 1994. p.42.

TORRES FILHO, J. \& SÁ, M. de F.P. Comportamento de linhagens de feijão-de-corda em relação à sarna. Anais, $1^{\circ}$ Seminário de Pesquisa Agropecuária - Agripesquisa, Fortaleza, CE. 1996. p.78-80.

TORRES FILHO, J. \& SOUZA, M.C.R. de. Reação de cultivares de caupi à sarna no planalto da Ibiapaba. Fortaleza. EPACE. Pesquisa em Andamento, 201996. 